\title{
Synthesis of Hurwitz Polynomial Families Using Root Locus Portraits
}

\author{
Alla Anatolyevna Nesenchuk ${ }^{1,2}$ \\ ${ }^{1}$ Department of the Digital Transformation Technique, United Institute of Informatics Problems of the Belarusian National Academy of \\ Sciences, Minsk, Belarus \\ ${ }^{2}$ Faculty of Information Technologies and Robotics, Belarusian National Technical University, Minsk, Belarus
}

\section{Email address:}

anes@newman.bas-net.by

\section{To cite this article:}

Alla Anatolyevna Nesenchuk. Synthesis of Hurwitz Polynomial Families Using Root Locus Portraits. Automation, Control and Intelligent Systems. Vol. 7, No. 3, 2019, pp. 84-91. doi: 10.11648/j.acis.20190703.12

Received: August 10, 2019; Accepted: September 16, 2019; Published: September 27, 2019

\begin{abstract}
The paper deals with the problem of synthesis of a stable characteristic polynomial families describing the control systems' dynamics in conditions of the interval uncertainty. Investigation is based on the system mathematical model in the form of its root locus portrait generated by the polynomial free term variation that is named in the paper as the "free root locus portrait". The root loci of the Kharitonov's polynomials family (subfamily) is picked out of the whole polynomial family and is considered for carrying out the investigation. Specific regularities of the interval root locus portrait have been discovered. On the basis of these regularities main properties of the system root locus portrait have been defined. A stability condition has been formulated that allows to calculate the polynomial free term variation interval ensuring the polynomial family hurwitz stability. This stability condition is applicable to the class of polynomials having their free root locus poles lying within the left half-plane of roots or, in other words, being stable when their free term is equal to zero. The stable family is being synthesized by setting up (adjusting) the given initial family that is supposed to be unstable, i.e. the proposed method of synthesis allows to turn stable (hurwitz) the given nonhurwitz interval polynomial family. The setting up criterion is specified in terms of proximity i.e. as the nearest distance from the "unstable" system roots to the "stable" ones as measured along the root trajectories. The stable polynomial could be selected as the nearest to the given unstable one with or without consideration of the system quality requirements. In the course of the setting up procedure new boundaries of only the polynomial free term variation interval (stability interval) are calculated that allows to ensure system stability without modification of its root locus portrait configuration. A numerical example of the polynomial setting up procedure has been given.
\end{abstract}

Keywords: Unstable Control System, Parametric Uncertainty, Interval Characteristic Polynomial, Parametric Synthesis, Root Locus, Kharitonov's Polynomials

\section{Introduction}

The issues of assuring the acceptable dynamic characteristics of the plants operation in conditions of uncertainty are currently among the most important ones that drive forward the development of the automatic control theory. $[1,2]$. In this area three basic directions of investigation could be singled out: algebraic, frequency and root locus one. Algebraic and root locus approaches both could be referred to the so-called "polynomial approach", the appearance of which dates back to the 1960 - 70th of the XX century, and which has been formed as the special approach to control systems design and synthesis [3]. In this connection in his book [3] V.
Kučera mentions that in many cases polynomial representation appears more natural.

The dynamic systems stable characteristic polynomials and polynomial families synthesis, analysis of the polynomial families stability, constituting one of the main directions of the parametric approach to robustness, represent today the complicated and important tasks $[4,5]$. Within the parametric approach to the problem the series of effective methods for analysis have been developed. One of the pioneer works in this direction was the paper of V. L. Kharitonov [6], where he proved that for the interval uncertain polynomial family asymptotic stability verification it is necessary and enough to check only four specific polynomials of the family with the 
definite combinations of constant coefficients. An analogue of Kharitonov theorem was formulated for the unstable interval polynomials' homogeneous classes of equivalence [7].

The algebraic approach [6] was then further developed in the series of works [7, 9-12], which comprise in particular the conditions of retaining strict hurwitz property (hurwitz robust stability criteria for polynomials with perturbed coefficients (polynomial families)) [9-11], i.e. the techniques were offered for calculating maximal possible deviations of the hurwitz characteristic polynomial coefficients (parameters) from their nominal values, which ensure retaining strict hurwitz property of the polynomial. The first work that appeared in this area was the paper of B. R. Barmish [9]. The task here was reduced to the single-parameter optimization problem. The close tasks were solved by Y. Soh in [10]. Conditions for the generalized stability of polynomials with the linearly dependent coefficients (polytopes) were obtained in paper [11] by G. Rantzer.

Based on the new interval polynomial stability criterion and Lyapunov theorem, a robust optimal proportional-integral-derivative (PID) controller is proposed in paper [12] to carry out design for different plants that contain perturbations of multiple parameters. A new stability criterion of the interval polynomial is presented to determine whether the interval polynomial belongs to Hurwitz polynomial or not.

In the works of Y. Z. Tsypkin and B. T. Polyak (see [4, 5, 8]) the frequency-domain approach to the polynomially described systems robustness was offered. The tasks, related to synthesis, were discussed in [13] and [14].

The root locus approach to robustness is considered in paper [15] by B. R. Barmish and R. Tempo, in the works of the author [16-20] and series of other works. Paper [15] deals with the method for synthesis of a compensator ensuring the required robust quality of uncertain plant. Solutions for the tasks of parametric synthesis and analysis of uncertain systems (interval dynamic systems (IDS) in particular) with application of the main provisions of the root locus theory are offered in [16-20].

However, the above mentioned techniques do not give the complete and general answer to the question about how do the given polynomial family coefficients' values should be changed to move the system roots (by some criteria) inside the given region in case when initially they do not get there. The existing methods in many cases are either too complicated and not easy for use or stuck to the specific application, and, therefore the field still keeps being opened for various creative solutions.

Substantial results obtained in the area of parametric approach to robustness are represented in the books [4, 5] by B T. Polyak, P. S. Scherbakov and M. V. Khlebnikov. In [4, 5] several tasks still finding their solutions are indicated and among them the task of searching the stable polynomial being the nearest to the given unstable one.

The contents of this paper are devoted to the root locus approach to the above described problem solution in application to the interval polynomial. It represents further development of works [16-20]. One of the main advantages of the root locus approach in application to uncertain systems investigation is that by its nature that approach implies variation of system parameters and allows to look at the system as if "from the inside" and to follow up how do the roots move in response to the parameters variation. The technique that has been developed can be used for setting up the values of the parameter variation intervals limits for ensuring the IDS stability in the cases when the stability test had shown that the given system was unstable.

\section{The Problem Formulation}

For getting more complete picture of the processes that occur in uncertain control systems it seems important to find correlation between algebraic, root locus and frequency methods of investigation. Such correlation exists and can be applied for finding dependence between the values of the system characteristic equation coefficients (parameters) variation and its dynamic properties and further determination of how and what specific coefficients should be altered for ensuring robust stability. A possible way of establishing the above mentioned correlation could be investigation of the systems root locus portraits versus Kharitonov's polynomials [6], which could be called root locus Kharitonov's polynomials.

Let us consider the IDS, described by the family of characteristic polynomials like

$$
F=\left\{P(s)=\sum_{j=0}^{n} a_{j} s^{n-j}=0\right\},
$$

where

$$
a_{j} \in\left[\underline{a}_{j}, \bar{a}_{j}\right]
$$

$\underline{a}_{0}>0, j=\overline{0, n}, \quad \underline{a}_{j}$ and $\bar{a}_{j}$ are correspondingly the lower and the upper bounds of the closed interval of uncertainty $\left[\underline{a}_{j}, \bar{a}\right], s=\sigma+i \omega$. The coefficients of polynomial (1) represent in fact the uncertain parameters.

Assume that the given family (1) is unstable. The task consists in synthesis of the stable interval family of polynomials on the basis of the given unstable one, i.e. for the case when checking stability of Kharitonov's polynomials gives a negative result. The "stable" intervals of the parameter variation assuring the family stability are calculated from the given intervals (2) so that they will have met the desirable dynamic characteristics of the system. The stability intervals definition criteria may in general be different. In our case in particular the criterion is specified in terms of proximity i.e. as the nearest distance from the "unstable" system roots to the "stable" ones as measured along the root trajectories.

\section{Free Root Locus Portrait as a Model of the Interval System}

Write an expression for any arbitrary subfamily $f$ (either continuous or not) of the polynomial family $F$ : 


$$
f \subset F .
$$

Introduce the series of definitions [16].

Definition 1. Name the root locus of the dynamic system characteristic equation (polynomial) as the dynamic system root locus.

Consider the family of the interval system root loci generated for the characteristic polynomials of (1).

Definition 2. Name the family (the set) of the interval dynamic system root loci as the root locus portrait of the interval dynamic system.

Definition 3. The algebraic equation coefficient or the parameter of the dynamic system, described by this equation, being varied in a definite way for generating the root locus, when it is assumed that all the rest coefficients (parameters) are constant, name as the free parameter of the algebraic equation root locus or simply the root locus parameter.

Definition 4. The algebraic equation root locus, which parameter is the coefficient $a_{k}$, name as the root locus relative to the coefficient $a_{k}$.

Definition 5. The root locus relative to the constant term of the dynamic system characteristic equation name as the free root locus of the dynamic system.

The peculiarity of the free root loci, which distinguishes them from another types of root loci, consists in the fact that all their branches strive to infinity along the corresponding asymptotes.

Definition 6. The points, from which the root locus branches initiate and where the root locus parameter is equal to zero, name as the root locus initial points.

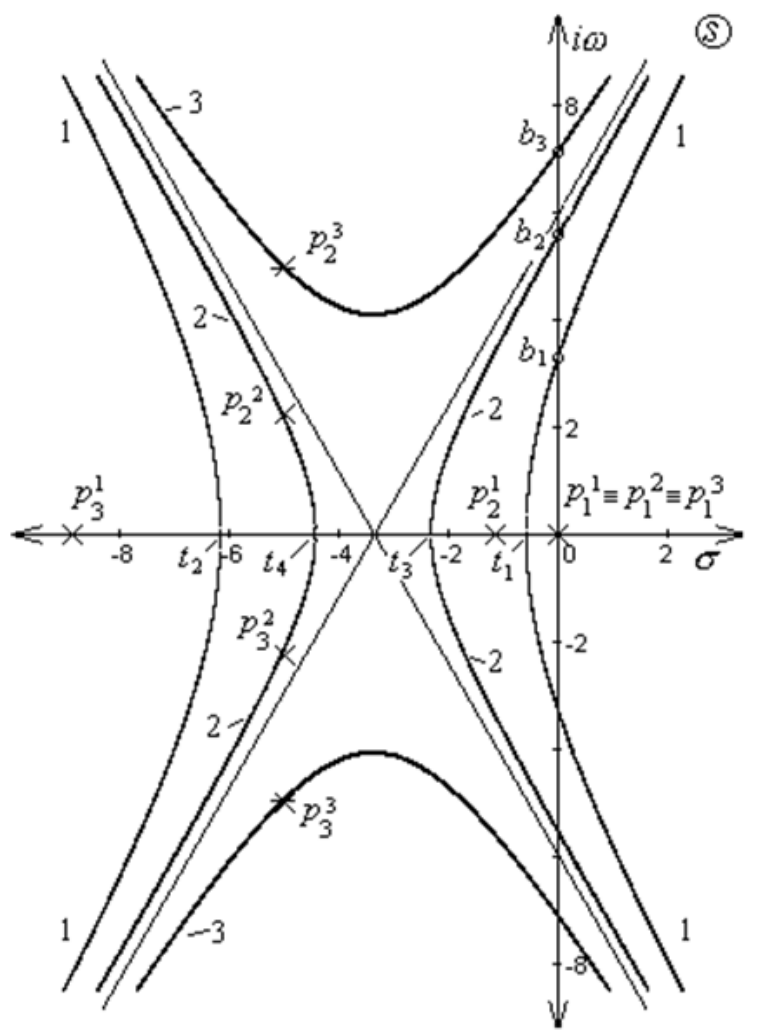

Figure 1. Root loci (subfamily) of the root locus portrait for the interval system of the third order.
Remark 1. One of the free root locus initial points is always located at the origin of the complex plane of roots $s$.

The above remark correctness follows from the form of equation (1). It is illustrated by Figure 1 where three root loci of the third order are represented. Root loci branches are indicated by the corresponding root locus sequential number: 1, 2, 3. Initial points in Figure 1 are depicted by X-s and symbols $p_{l}^{r}$ with two indexes, of which the lower one means the initial point sequential number, $l=1,2,3$, and the upper the number of the root loci, $r=1,2,3$. From Figure 1 it is evident that one initial point of every root loci is located at the origi: $p_{1}^{1} \equiv p_{1}^{2} \equiv p_{1}^{3}$.

Remark 2. The real positive branch of the free root locus, which begins at the initial point located at the origin, always strives along the negative real semi-axis $\sigma$ of the complex plane and is directed to the left half-plane.

Remark 2 is correct because real roots of equations with positive coefficients are always negative and also over the root loci properties [1].

Remark 2 is confirmed by Figure 1. In this figure the real portion $\left[t_{1}, p_{1}^{1}\right]$ of the root locus 1 branch, located between points $p_{1}^{1}$ and $\mathrm{t} 1$, is directed from the initial point $p_{1}^{1}$ to point $t 1$ and then along the right branch 1 to the point $b 1$ and further to infinity. The same feature holds for root loci 2 and 3 . The real portion $\left[t_{3}, p_{1}^{2}\right]$ of the root locus 2 branch, located between points $p_{1}^{2}$ and $t_{3}$, is directed from the origin to the left half-plane.

For carrying out investigation apply the Teodorchik - Evans free root loci (TEFRL). The Teodorchik - Evans root locus [16] means the root locus generated by the root locus parameter $a_{k}$ variation within the interval $-\infty<a_{k}<+\infty$ of the real values. Thus, hereinafter the term "root locus" means the TEFRL, and its root locus parameter represents the constant term of the system characteristic polynomial.

To generate the IDS root locus portrait apply the family of the mapping functions

$$
s^{n}+a_{1} s^{n-1}+\ldots+a_{n-1} s=u(\sigma, \omega)+i v(\sigma, \omega)=-a_{n},
$$

obtained from (1), where $u(\sigma, \omega)$ and $v(\sigma, \omega)$ are harmonic functions of two independent real variables $\sigma$ and $\omega ; a_{n}$ is the root locus parameter. Analytical and graphical root loci are formed using mapping function (4). The root locus equation is as follows:

$$
\mathrm{v}(\sigma, \omega)=0,
$$

and the parameter equation [16] as follows:

$$
u(\sigma, \omega)=-a_{\mathrm{n}} .
$$

The fragmentary root locus portrait [16] for the IDS of the forth order made up of four Kharitonov's polynomials free root loci is shown in Figure 2. The Kharitonov's polynomials $h_{1}, h_{2}, h_{3}$ and $h_{4}$ in this figure are represented by points (polynomial roots) marked with circles, white squares, triangles, and black squares correspondingly. Branches, 
belonging to the root locus of the definite polynomial, are designated similar to the corresponding polynomial, i.e. $h_{1}, h_{2}$, etc. The following designations are accepted in Figure 2: symbols $c_{1}$ and $c_{2}$ indicate the asymptotes cross centers for the root loci of polynomials $h_{i}$; points $b_{i}, i \in\{1,2,3,4\}$, represent the cross points of the root loci branches, located in the upper half-plane, with the system asymptotic stability boundary, axis $i \omega ; \alpha_{q}, q=\overline{1,8}$, are the root loci asymptotes. The root loci initial points, which represent zeroes of mapping function (4), are depicted by X-s and the letters $p_{l}^{r}$ of which the lower index $l$ means the definite root locus initial point sequential number and the upper one $r$ is the sequential number of the corresponding polynomial (root locus), $l=\overline{1,4}, \quad r=\overline{1,4}$. Initial points with number "1" ( $\left.p_{1}^{r}\right)$ coincide at the origin, $p_{1}^{1} \equiv p_{1}^{2} \equiv p_{1}^{3} \equiv p_{1}^{4}$.

From Figure 2 it is evident that two complex-conjugate roots of each one of the Kharitonov's polynomials are located in the right half-plane. Therefore, the given interval system is asymptotically unstable [6].

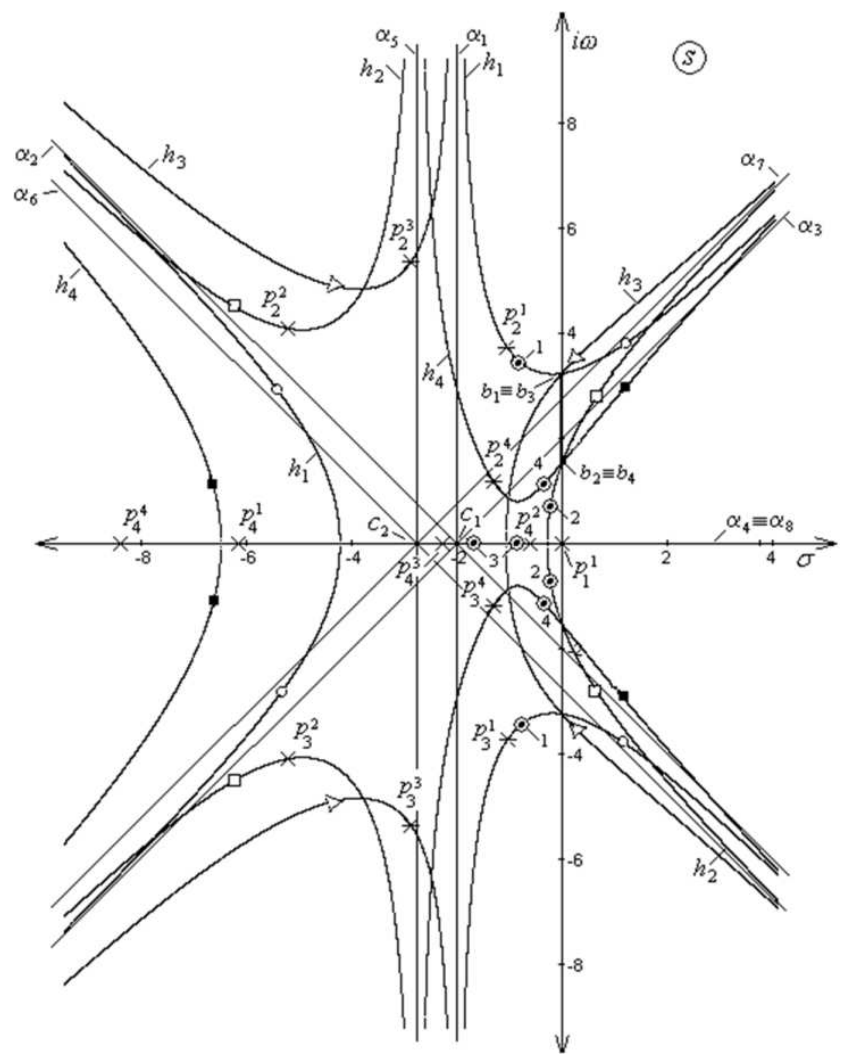

Figure 2. Root loci of Kharitonov's polynomials for the system of the fourth order.

\section{The Uncertain System Root Locus Portrait Dynamics Investigation}

Those branches of the IDS free root locus portrait that cross the stability boundary, i.e. the axis $i \omega$ of the complex plane $s$, generate on this axis a region (set) of cross points. Let's name this region as the cross region and designate it as $R_{\omega}$. Along the complex variable theory and over the complex mapping function (4) continuity property this region $R_{\omega}$ represents a many-sheeted region and is composed of the separate sheets with every sheet (a continuous subregion) formed by a separate root locus branch as it moves in the complex plane following the parameters variation. The cross region portion generated by only positive branches of the system root locus portrait name as the positive cross region and designate it as $R_{\omega}^{+}$,

$$
R_{\omega}^{+} \subset R_{\omega}
$$

Specify also some subregion $r_{\omega}^{+}$(either continuous one or not) of the cross region $R_{\omega}{ }^{+}$(7) generated by the root loci branches of any arbitrary subfamily (subset) $f$ of the interval system polynomial family (1), and name it as the (positive) cross subregion. Hence,

$$
r_{\omega}^{+} \subset R_{\omega}^{+}
$$

In Figure 2 the subregion $r_{\omega}^{+}$is formed by the brunches of the Kharitonov's polynomials and lies between points $b_{1}$ and $b_{2}$, i.e. $r_{\omega}^{+}=b_{1} b_{2}$.

Then introduce the following sets:

$$
\begin{aligned}
& W_{r}^{+}=\left\{\omega_{r_{i}}^{+}\right\}, \\
& A_{r}^{+}=\left\{a_{r_{i}}^{+}\right\},
\end{aligned}
$$

where $W_{\mathrm{r}}^{+}$is the set (family) of coordinates $\omega_{r_{i}}^{+}$of points forming the cross subregion $r_{\omega}{ }^{+}(8) ; A_{r}^{+}$is the set (family) of values $a_{r_{i}}^{+}$of the root locus parameter $a_{n}$ at the corresponding points forming the set $W_{r}^{+}$.

Now define the minimal value $a_{r \text { min }}^{+}$of the root locus parameter within the cross subregion $r_{\omega}{ }^{+}$:

$$
a_{r \min }^{+}=\inf A_{r}^{+} \text {. }
$$

Peculiarities of the IDS root loci branches initial points location make it possible to conclude about existence of its characteristic equation coefficient variation intervals ensuring asymptotic stability of the given system.

Statement. If the initial points of the interval polynomial subfamily $f(3)$ free root loci (except the points always situated at the origin) are located in the left half-plane $s$, there exists the maximum possible interval $d$ of the root locus parameter $a_{n}$ values,

$$
d=\left(0, a_{r \min }^{+}\right),
$$

that ensures the asymptotic stability of the subfamily $f$.

Proof. The subfamily $f$ free root loci generate at the system stability boundary, axis $i \omega$, the cross subregion $r_{\omega}{ }^{+}(8)$ of cross points (see Figure 2), which corresponds to some subsets from the set $W_{r}^{+}(9)$ of these cross points coordinates and the set $A_{r}^{+}$ 
(10) of the parameter values at these cross points. Due to the statement precondition that the initial points of the given subfamily $f$ free root loci are located in the left half-plane it can be stated that on every $i$-th branch of these root loci, which crosses the axis $i \omega$, there exists an interval (an interval set) of roots,

$$
S_{i}=\left(p_{i}, b_{i}\right)
$$

lying between the $i$-th branch initial point $p_{i}$ (see points $p_{l}^{r}$ : $p_{2}{ }^{1}, p_{3}{ }^{1}, p_{2}{ }^{4}, p_{3}{ }^{4}, p_{1}{ }^{1}, p_{4}{ }^{2}, p_{4}{ }^{3}$ in Figure 2) and the cross point $b_{i}$ (points $b_{1}, b_{2}, b_{3}, b_{4}$ in Figure 2) of this branch with the stability boundary $i \omega$ that is completely located in the left half-plane. Real coordinates $\sigma$ of interval (13) points lay within the corresponding interval

$$
\Omega_{i}=\left(\sigma_{p_{i}}, \sigma_{b_{i}}\right)=\left(\sigma_{p_{i}}, 0\right),
$$

where $\sigma_{p_{i}}$ is the coordinate $\sigma$ at the initial point $p_{i}$, and $\sigma_{b_{i}}$ is the coordinate $\sigma$ at the cross point $b_{i}$. The corresponding root locus parameter $a_{n}$ values for points of interval (13) lie within the interval

$$
A_{n_{i}}=\left(a_{p_{i}}, a_{r}^{+}{ }_{i}\right)=\left(0, a_{r}^{+}{ }_{i}\right),
$$

where $a_{p_{i}}$ is the value of $a_{n}$ at the initial point $p_{i}$, and $a_{r_{i}}^{+}$ see in (10). Root locus parameter values within (15) guarantee asymptotic stability of the corresponding $i$-th root loci branch. Intervals $A_{n_{i}}$ for the whole subfamily $f$ constitute the set $A_{n}$ of the root locus parameter intervals:

$$
A_{n}=\left\{A_{n_{i}}\right\}=\left\{A_{1}, A_{2}, \ldots, A_{i}, \ldots\right\}=\left\{\left(0, a_{r_{i}}^{+}\right)\right\},
$$

which ensures asymptotic stability of the whole subfamily $f$, and intervals (14) for the whole subfamily constitute the set

$$
\Omega=\left\{\Omega_{i}\right\}=\left\{\left(\sigma_{p_{i}}, \sigma_{b_{i}}\right)\right\}=\left\{\left(\sigma_{p_{i}}, 0\right)\right\},
$$

which includes intervals of real coordinates $\sigma$ corresponding to the intervals of set (16).

The minimal element $A_{n_{d}}$ of $A_{n}\left(A_{n_{d}} \in A_{n}\right)$,

$$
A_{n_{d}}=\inf A_{n}=\left(0, a_{r_{\text {min }}}^{+}\right),
$$

obtained on the base of (11), (15) and (16), name the parameter dominating interval, and the branch, on which this interval is located, name the dominating branch. The interval $\sigma_{d} \in \Omega$ corresponding to the dominating interval (18) is determined from (17) as

$$
\sigma_{d}=\left(\sigma_{p_{d}}, \sigma_{b d}\right)=\left(\sigma_{p_{d}}, 0\right),
$$

where $\sigma_{p_{d}}$ and $\sigma_{b_{d}}$ are coordinates $\sigma$ of points corresponding to the dominating interval minimum and maximum limit values. So, the expression for the intersection of sets $A_{n_{i}}$ :

$$
A_{n_{c}}=\bigcap_{A_{n i} \in A_{n}} A_{n_{i}}=\inf A_{n}=A_{n_{d}}=\left(0, a_{r_{\text {min }}}^{+}\right)=d
$$

gives us the maximal possible interval ensuring the system stability. Interval (20) is similar to interval (12) that proves existence of interval (12). Interval (12) is the maximum possible one also because it is similar to the dominating interval (18), and for this reason on the base of (19) it can be stated that $a_{n} \geq a_{r_{\text {min }}}^{+} \rightarrow \sigma_{b_{d}} \geq 0$ that completely proves the statement.

It should be mentioned here that the initial points located at the origin (see Remark 1) do not have an influence upon stability because, in accordance with Remark 2, the positive real branches initiating at this points within their vicinity are always directed along the negative real axis to the left half-plane.

Definition 7. The maximal possible interval of polynomial (1) root loci parameter values ensuring the polynomial asymptotic stability property name the polynomial stability interval (by this parameter) or the polynomial dominating interval.

When some root locus initial points are located at the stability boundary (except the points that are always located at the origin), and on the assumption that all the rest points are located in the left half-plane, the additional analysis is required for finding the stability interval existence. The analysis implicates necessity to define the root loci branches direction in the vicinity of the initial points, located at the stability boundary, i.e. just to determine what half-plane they are directed to: left one or right one. Such stability interval exists in the following cases:

a) all the root locus branches, starting at initial points located on the stability boundary, are directed from these points to the left half-plane;

b) all positive root loci branches, starting at initial points located on the stability boundary, are directed from these points to the left half-plane.

For determination of the above indicated branches direction at the initial points it is enough to define the root locus sensitivity vector [16] direction at this points.

The IDS root locus portraits analysis that has been carried out made it possible to find several general regularities being inherent in the Kharitonov's polynomials free root loci:

1. paired convergence of the root loci branches for the pair $h_{1}(s)-h_{3}(s)$ and pair $h_{2}(s)-h_{4}(s)$ at the complex plane imaginary axis (points $b_{1}, b_{2}, b_{3}, b_{4}$ in Figure 2);

2. paired convergence of the corresponding asymptotes centers for the pair $h_{1}(s)-h_{3}(s)$ and pair $h_{2}(s)-h_{4}(s)$ at the real axis of the complex plane at points $c_{1}$ and $c_{2}$ (see in Figure 2);

3. the tendency for the system robust properties variation while varying its characteristic polynomial coefficients values.

The results obtained provide the possibility to determine existence of the system characteristic equation coefficients' 
variation intervals ensuring its robust stability and also to define how the coefficients values should be changed for the system dynamic characteristics correction if it is required. The obtained results hold for the whole interval family (1).

The IDS root locus portraits investigation, which has been carried out, confirms that they can be successfully applied for the in-depth studying robust properties for systems of this kind.

\section{An Algorithm for the Stable Uncertain Families Synthesis}

The previous section provided us with the statement for checking existence of the polynomial family (1) coefficients stability intervals. In this section we define what the values of these intervals should be. For this purpose consider polynomial subfamily (3), consisting of the IDS Kharitonov's polynomials, and develop the procedure for synthesis of the stable Kharitonov's polynomials on the base of the given unstable ones, which depends on the system root loci initial points location in relation to the asymptotic stability boundary $i \omega$. For the synthesis procedure development apply the Kharitonov's polynomials free root loci. Consider the case, when initial points are located in the left half-plane. In this case the algorithm of synthesis could be represented by the following stages.

Stage 1. Obtaining the TEFRL analytical equation (5) for each one of the IDS four Kharitonov's polynomials.

As the Kharitonov's polynomials represent the subfamily $f$ (3) of the IDS polynomial family, they generate the above described cross subregion $r_{\omega}^{+}(8)$ on the stability boundary, which is formed by the set $W_{r}^{+}$(9) of the cross points coordinates.

Stage 2. Calculating coordinates $\omega_{r_{i}}^{+}$of the set (9) at points $b_{i}$ (see Figure 2 ) by solution of the TEFRL equations, obtained in stage 1 , relative to $\omega$ in condition when $\sigma=0$. In this way the set $W_{r}^{+}(9)$ is formed.

For every obtained value of $\omega_{r_{i}}^{+}$of $W_{r}^{+}$the corresponding value $a_{r_{i}}^{+}$of the root locus parameter $a_{n}$ is calculated by formula (6) thus forming the set $A_{r}^{+}$(10).

Stage 3. Determination of the stability (dominating) interval by the coefficient $a_{n}$.

For this purpose, using (11), define the minimal one, $a_{r \text { min }}^{+}$, of the parameter values at points of the set $A_{r}^{+}$. Thus, obtain the stability interval $d$ (12) of the parameter $a_{n}$ variation, which ensures stability of the Kharitonov's polynomials and, therefore, the system in whole. Formulate the following theorem.

Stability condition. For ensuring robust stability of the polynomial family (1) it is necessary and sufficient to ensure that the upper limit of the constant term $a_{n}$ variation gets within the interval

$$
0<\bar{a}_{n}<a_{r \min }^{+}
$$

if the family is stable when $a_{n}=0$.

Proof. Let the coefficient $a_{n}$ to be the polynomial family (1) root locus parameter. Along the stability condition family (1) is stable when $a_{n}=0$, i.e. its root loci initial points are located in the left half-plane. In such case in accordance with the above statement the family is asymptotically stable when the root locus parameter values lay within interval (12). Therefore, in view of the above statement the stability condition is correct.

Stage 4. Comparing stability interval (21), obtained on the previous stage, with the given interval $a_{n} \in\left[\underline{a}_{n}, \bar{a}_{n}\right]$ of the parameter $a_{n}$ variation and altering the system stability in correspondence with inequality (21). In case if condition (21) is not satisfied the upper limit $\bar{a}_{n}$ of the constant term $a_{n}$ variation interval is set up in accordance with this inequality (21).

When the power $n$ of the IDS characteristic polynomial is less than or equal to $3, n \leq 3$, the above given stability condition can be applied without any preconditions i.e. for applying the stability condition it is not required to satisfy the asymptotic stability precondition of the given family when $a_{n}$ $=0$, because in this case the polynomial coefficients positivity always guarantees negativity of all the roots real parts.

The above described algorithm allows to carry out the parametric synthesis of the stable interval system without modification of its root locus portrait configuration i.e. only by the simple procedure of setting up the characteristic polynomial constant term variation interval limits.

\section{A Numerical Example}

Consider a numerical example, demonstrating the results obtained.

Describe the interval system by the characteristic polynomial like

$$
\mathrm{s}^{4}+a_{1} s^{3}+a_{2} s^{2}+a_{3} s+a_{4}=0
$$

where the real coefficients vary within the following intervals: $a_{0}=1 ; 8 \leq a_{1} \leq 11 ; 25 \leq a_{2} \leq 50 ; 26 \leq a_{3} \leq 84 ; 550 \leq a_{4} \leq 560$.

Consider the coefficient $a_{4}$ to be the root locus parameter (see Definition 3). Define the mapping function (4) (root locus equation in the general form) for the given system:

$$
\begin{gathered}
-a_{4}=a_{0} \sigma^{4}+4 a_{0} \sigma^{3} i \omega-6 a_{0} \sigma^{2} \omega^{2}-4 a_{0} \sigma i \omega^{3}+a_{0} \omega^{4}+a_{1} \sigma^{3}+3 a_{1} \sigma^{2} i \omega- \\
-3 a_{1} \delta \sigma^{2}-a_{1} i \omega^{3}+a_{2} \sigma^{2}+2 a_{2} \delta i \sigma-a_{2} \omega^{2}+a_{3} \sigma+a_{3} i \omega .
\end{gathered}
$$

Then proceed along the corresponding stages of the algorithm given in section 5 .

Stage 1. Define correspondingly the TEFRL (5) and the parameter (6) equations:

$$
\begin{gathered}
\omega\left(4 a_{0} \sigma^{3}-4 a_{0} \sigma \omega^{2}+3 a_{1} \sigma^{2}-a_{1} \omega^{2}+2 a_{2} \sigma+a_{3}\right)=0 ; \\
a_{0} \sigma^{4}-6 a_{0} \sigma^{2} \omega^{2}+a_{0} \omega^{4}+a_{1} \sigma^{3}-3 a_{1} \sigma \omega^{2}+a_{2} \sigma^{2}-a_{2} \sigma=-a_{4} .
\end{gathered}
$$


These equations are required correspondingly to generate the root locus curves and calculate the parameter values at any point of these curves.

Specify the Kharitonov's polynomials [6] for the interval system described by (22):

$$
\begin{aligned}
& h_{1}(s)=s^{4}+8 s^{3}+25 s^{2}+84 s+560 \\
& h_{2}(s)=s^{4}+11 s^{3}+50 s^{2}+26 s+550 \\
& h_{3}(s)=s^{4}+8 s^{3}+50 s^{2}+84 s+550 \\
& h_{4}(s)=s^{4}+11 s^{3}+25 s^{2}+26 s+560
\end{aligned}
$$

Free root loci of these polynomials are represented in Figure 2 described above (see section 3 ).

Number of asymptotes $n_{a}$ (in Figure 2 the asymptotes are indicated as $\left.\alpha_{1}, \alpha_{2}, \ldots, \alpha_{8}\right)$ is constant for each one of Kharitonov's polynomials and is equal to $n_{a}=n-m=4-0=4$, where $m$ is the number of poles of the function (4). The centers of asymptotes are located on the axis $\sigma$ and have the following coordinates: $c_{1}=2.0 ; c_{2}=2.7$ (see Figure 2). The asymptotes and their centers' coordinates coincide in pairs: for the pair $h_{1}(s)-h_{3}(s)$ and for the pair $h_{2}(s)-h_{4}(s)$ the root loci asymptotes centers are located correspondingly at point $c_{1}$ and point $c_{2}$; the root loci branches of the polynomial pair $h_{1}(s)-$ $h_{3}(s)$ strive to the same asymptotes $\alpha_{1}, \alpha_{2}, \alpha_{3}$ and $\alpha_{4}$ and of the pair $h_{2}(s)-h_{4}(s)$ - to the asymptotes $\alpha_{5}, \alpha_{6}, \alpha_{7}$ and $\alpha_{8}$.

The inclination angles of asymptotes for the given root loci are correspondingly the following:

$\varphi_{1}=0^{\circ}$ (for asymptotes $\alpha_{4}$ and $\alpha_{8}$ ); $\varphi_{2}=45^{\circ}$ (for asymptotes $\alpha_{3}$ and $\left.\alpha_{7}\right)$;

$\varphi_{1}=90^{\circ}$ (for asymptotes $\alpha_{1}$ and $\alpha_{5}$ ); $\varphi_{1}=135^{\circ}$ (for asymptotes $\alpha_{2}$ and $\alpha_{6}$ ).

The cross subregion $r_{\omega}^{+}$(8) for the given interval subfamily is defined as $r_{\omega}^{+}=\left[b_{2}, b_{1}\right]$ (see it in Figure 2).

Stage 2. Obtain the set $W_{r}^{+}=\{3.24,1.537,3.24,1.537\}$ of coordinates $\omega_{r_{i}}^{+}$(see (9)) correspondingly for points $b_{1}(0$; $3.24), b_{2}(0 ; 1.537), b_{3}(0 ; 3.24), b_{4}(0 ; 1.537)$.

Calculate using formulas (4) and (6) and define the following values of root locus parameter $a_{r_{i}}^{+}$for the set $A_{r}^{+}$ (10) at points $b_{1}, b_{2}, b_{3}$ and $b_{4}$ where the corresponding Kharitonov's polynomials' root loci branches cross the imaginary axis:

$$
\begin{aligned}
& a_{r_{1}}^{+}=152.3 \text { for polynomial } h_{1} \text { at point } b_{1} ; \\
& a_{r_{2}}^{+}=112.6 \text { for polynomial } h_{2} \text { at point } b_{2} ; \\
& a_{r_{3}}^{+}=414.8 \text { for polynomial } h_{3} \text { at point } b_{3} ; \\
& a_{r_{4}}^{+}=53.5 \text { or polynomial } h_{4} \text { at point } b_{4} .
\end{aligned}
$$

Thus, the set $A_{r}^{+}$has been formed: $A_{r}^{+}=\{152.3,112.6$, $414.8,53.5\}$.

Stage 3. For calculating the dominating interval (12), which ensures the system stability, the minimal value (11) of the root locus parameter within the cross subregion is defined as per the algorithm:

$$
a_{r \min }^{+}=\inf A_{r}^{+}=a_{r 4}^{+}=53.5 .
$$

Therefore, the stability (dominating) interval (12) is defined as

$$
d=\left(0, b_{4}\right)=(0,53.5)
$$

Thus, according the stability condition the given system is asymptotically stable when the following inequality holds:

$$
0<\bar{a}_{4}<53.5 \text {. }
$$

Stage 4. To define the equation (22) coefficient intervals, ensuring the system stability, the stability condition (21) is applied. As in (22) the coefficient $\bar{a}_{4}>53.5$, then in correspondence with (21) the given interval system is asymptotically unstable. It is clearly understandable from Figure 2, where it is evident that the roots (designates in Figure 2 as triangles, squares, circles and filled squares) of Kharitonov's polynomials are located in the right half-plane. Therefore, the parameter $a_{4}$ upper limit should be adjusted in accordance with the stability condition inequality (24) and dominating interval (23). Thus, it should be as follows:

$$
\bar{a}_{4}<53.5 \text {. }
$$

The calculated "stable" locations of the Kharitonov's polynomials roots for the case when $\bar{a}_{4}=30$ are designated on the root loci branches, crossing the stability boundary, by encircled points with digits that corresponds to the numbers of the appropriate polynomials, e.g. for polynomial $h_{1}$ there are four roots indicated by digit 1 (see Figure 2). From Figure 2 it is evident that all these roots of Kharitonov's polynomials in this case are located in the left half-plane.

\section{Conclusion}

The task of attaining stability of the interval family of control system characteristic polynomials has been solved in the paper. The initially unstable (nonhurwitz) family is converted stable (hurwitz) by specifically setting up the free term of the family. For the task solution the investigation of the system root locus portrait behavior at the asymptotic stability boundary has been carried out by analyzing the family's Kharitonov's polynomials root locus portrait. On this basis the system robust stability condition has been formulated. The method and algorithm have been developed for setting up the interval polynomial so that it gets stable in cases when the stability verification showed that the initial polynomial was unstable. If the system order is $n>3$, this stability condition is applicable to the class of polynomials having their free root locus poles lying within the left half-plane of roots or, in other words, being stable when their free term is equal to zero. If $n \leq$ 3 , the method is applied without any conditions (limitations). The algorithm considered allows to carry out parametric synthesis of the stable interval family without its root locus 
portrait configuration modification, i.e. by only setting up the limit values of the characteristic polynomial coefficient variation intervals. In this way the stability interval for the initially unstable polynomial is defined. The setting up criterion is specified in terms of proximity i.e. as the nearest distance from the "unstable" system roots to the "stable" ones when measured along the root trajectories. The stable polynomial could be then selected as the nearest to the given unstable one with or without consideration of the system quality requirements. A numerical example of the polynomial setting up procedure has been given in the paper.

\section{Acknowledgements}

The work was supported by a grant of the Belarusian Republican Fund of Fundamental Research.

\section{References}

[1] R. C. Dorf and R. H. Bishop, Modern Control Systems, 12th ed. New York: Prentice Hall, 2011, 1084.

[2] R. Tempo, C. Calafiori, and F. Dabbene, Randomized Algorithms for Analysis and Control of Uncertain Systems With Applications. London: Springer-Verlag, 2013, 357.

[3] V. Kučera, "Polynomial control: past, present, and future," International Journal of Robust and Nonlinear Control, vol. 17, no. 8, pp. 682-705, May 2007.

[4] B. T. Polyak and P. S. Scherbakov, Robust Stability and Control [in Russian]. Moscow: Nauka, 2002, 303.

[5] B. T. Polyak, M. V. Khlebnikov, and P. S. Shcherbakov, Linear Systems Control in Presence of External Perturbations [in Russian]. Moscow: LENAND, 2014, 560.

[6] V. L. Kharitonov, "Asymptotic stability of an equilibrium position of a family of systems of linear differential equations," Differential Equations, vol. 14, pp. 1483-1485, 1979.

[7] V. Dikusar, G. Zelenkov, and N. Zubov, "Criteria of existence of homogeneous classes of equivalence for unstable interval polynomials" [in Russian], Doklady AN, Control theory, vol. 429, pp. 322-324, 2009.

[8] B. T. Polyak, Y. Z. Tsypkin, "Frequency criteria of robust stability and aperiodicity of linear systems,"Automation and Remote Control, vol. 51, no. 9 pt 1, pp. 1192-1201, September 1991.

[9] B. R. Barmish, "Invariance of the strict hurwitz property for polynomials with perturbed coefficients," IEEE Trans Automat Control, vol. 29, pp. 935-936, October 1984.
[10] Y. Soh, "Strict hurwitz property of polynomials under coefficient perturbations," IEEE Trans Automat Control, vol. 34, pp. 629-632, June 1989.

[11] A. Rantzer, "Stability conditions for polytopes of polynomials," IEEE Trans Automat Control, vol. 37, pp. 79-89, Janiary 1992.

[12] X. Li, H. Yu, M. Yuan, and J. Wang, "Design of robust optimal proportional-integral-derivative controller based on new interval polynomial stability criterion and Lyapunov theorem in the multiple parameters' perturbations circumstance," IET Control Theory \& Applications, vol. 4, pp. 2427-2440, November 2010 .

[13] E. N. Gryazina, B. T. Polyak, "Stability regions in the parameter space: D-decomposition revisited," Automatica, vol. 42, pp. 13-26, January 2006.

[14] A. V. Kraev, A. S. Fursov, "Estimating the instability radii for polynomials of the arbitrary power" [in Russian], in Nonlinear Dynamics and Control, issue 4, S. V. Emelyanov and S. K. Korovin, Eds. Moscow: FIZMATLIT, 2006, pp 127-134.

[15] B. R. Barmish, R. Tempo, "The robust root locus," Automatica, vol. 26, pp. 283-292, February 1990.

[16] A. A. Nesenchuk,"Analysis and Synthesis of Robust Dynamic Systems on the Basis of the Root Locus Approach" [in Russian], Minsk: United Institute of Informatics Problems of the Belarusian National Academy of Sciences, 2005, 234.

[17] A. A. Nesenchuk, "Parametric synthesis of qualitative robust control systems using root locus fields," in Proceedings of the 15th Triennial World Congress of IFAC, Robust Control, vol. E, F. Camacho, L. Basanez, and J. A. de la Puente, Eds. Oxford: Elsevier Science, 2003, pp. 331-335.

[18] A. A. Nesenchuk, "The root locus method of synthesis of stable polynomials by adjustment of all coefficients," Automation and Remote Control, vol. 71, pp. 1515-1525, August 2010.

[19] A. A. Nesenchuk, "Investigation of behavior and synthesis of interval dynamic systems' characteristic polynomials based on the root locus portrait parameter function" [Electronic resource], Proceedings of the 60th American Control Conference (ACC 2018), Milwaukee, USA, pp. 2041-2046, 2018. Mode of access: http://www.proceedings.com/40219.html, date of access: 08.07.2019.

[20] A. A. Nesenchuk, "Investigation and synthesis of robust polynomials in uncertainty on the basis of the Root Locus Theory," in Polynomials - Theory and Applications, Cheon Seoung Ryoo, Ed. London: Intechopen, 2019, ch. 6, pp. 109130. 